\title{
Defective urinary carnitine transport in heterozygotes for primary carnitine deficiency
} Fernando Scaglia, MD, Yuhuan Wang, MS, Rani H. Singh, PhD, Philip P. Dembure, PhD,
Marzia Pasquali, PhD, Paul M. Fernhoff, MD, and Nicola Longo, MD, PhD

Purpose: Primary carnitine deficiency is an autosomal recessive disorder caused by defective carnitine transport and manifests as nonketotic hypoglycemia or skeletal or heart myopathy. Methods: To define the mechanisms producing partially reduced plasma carnitine levels in the parents of affected patients, we examined carnitine transport in vivo and in the fibroblasts of a new patient and his heterozygous parents. Results: Kinetic analysis of carnitine transport in fibroblasts revealed an absence of saturable carnitine transport in the proband's cells and a partially impaired carnitine transport in fibroblasts from both parents, whose cells retained normal $K_{m}$ values toward carnitine $(6-9 \mu \mathrm{M})$ but reduced $V_{\max }$. At steady state, normal fibroblasts accumulated carnitine to a concentration that was up to 80 times the extracellular value 0.5 $\mu \mathrm{M})$. By contrast, cells from the proband had minimal carnitine accumulation, and cells from both parents had intermediate values of carnitine accumulation. Plasma carnitine levels were slightly below normal in both heterozygous, yet clinically normal, parents and in the paternal grandfather and the maternal grandmother. To define the mechanism producing partially decreased carnitine levels, we studied urinary carnitine losses in heterozygous parents compared with controls. Urinary losses increased linearly $(P<0.05)$ with plasma carnitine levels in normal controls. When urinary carnitine losses were normalized to plasma carnitine levels, a significant difference was observed between controls and heterozygous individuals

$(P<0.01)$. Conclusions: These results indicate that fibroblasts from heterozygotes for primary carnitine deficiency have a decreased capacity to accumulate carnitine and that heterozygotes have increased urinary losses, which may contribute to their reduced plasma carnitine levels.

Key words: Carnitine, fatty acid oxidation, membrane transport, renal resorption

From the Division of Medical Genetics, Department of Pediatrics, Emory University, Atlanta, Georgia

Address correspondence to: Nicola Longo, $M D$, PhD, Division of Medical Genetics, Department of Pediatrics, Emory University, 2040 Ridgewood Drive, Atlanta, GA 30322. E-mail: nl@rw.ped.emory.edu

(91998 Genetics in Medicine. All rights reserved. 1098-3600\$0.00

\section{INTRODUCTION}

Primary carnitine deficiency (OMIM \#212140) is a rare autosomal recessive disorder caused by defective carnitine transport. Affected children present early in life with nonketotic hypoglycemia or at 2 to 3 years of age with skeletal or heart myopathy. Their plasma carnitine levels are markedly reduced (less than $10 \%$ of controls) and there are no consistently abnormal organic acids in the urine, excluding secondary carnitine deficiency. ${ }^{\prime}$ Affected patients waste carnitine in urine and their fibroblasts share the defective carnitine transporter with the kidney. ${ }^{2,3}$ This disorder responds promptly to dietary carnitine supplementation, which results in correction of metabolic abnormalities and even reversal of skeletal and heart muscle abnormalities. ${ }^{1}$ More than 20 children with this disorder have been reported. ${ }^{4-8}$ In several families, undiagnosed siblings of affected patients have died of hypoglycemia, sudden infant death syndrome, or intractable cardiomyopathy. ${ }^{1}$ Heterozygotes for this condition have partially reduced plasma carnitine levels, although the mechanism at the basis of this phenomenon remains unclear. In one family, partial carnitine deficiency (levels reduced to about $50 \%$ of controls) in the heterozygous brother of an affected patient has been associated with hypertrophic cardiomyopathy, similar to but less severe than that seen in homozygous patients. ${ }^{9}$ It is not known if such a presentation in the heterozygote was related to partial carnitine deficiency and impairment of fatty acid oxidation.

Carnitine can be synthesized by the human body or obtained from diet. Significant carnitine synthesis from lysine and methionine has been demonstrated in liver and kidneys, but not in heart or skeletal muscles, which depend on carnitine transport for fatty acid oxidation. ${ }^{1}$ Carnitine is present mostly in beef meat $(5.5 \mu \mathrm{mol} / \mathrm{g})$, with reduced amounts in pork $(1.5 \mu \mathrm{mol} / \mathrm{g})$ and chicken $(0.2 \mu \mathrm{mol} / \mathrm{g})$. Dairy products contain some carnitine $(0.2 \mu \mathrm{mol} / \mathrm{mL}$ in whole milk), whereas fruits and vegetables (excluding asparagus) contain negligible amounts $(<0.005$ $\mu \mathrm{mol} / \mathrm{g}) .^{10}$ Carnitine is lost in urine and also is secreted in the bile, where millimolar concentrations of carnitine conjugates are present and are clinically useful for the postmortem diagnosis of sudden infant death caused by inherited disorders of fatty acid oxidation. ${ }^{11}$ The quantitative contribution of biliary excretion to total carnitine losses in humans remains unclear. ${ }^{11}$ In healthy individuals, consumptions of very limited amounts of carnitine, as in strict vegetarians, does not result in severe carnitine deficiency. 
However, there is a report of fulminant neonatal death in a child with primary carnitine deficiency born to a heterozygous vegetarian mother, ${ }^{12}$ confirming the interaction of the diet with the genetic background in precipitating this inherited condition. It is not known if the association of a lowcarnitine diet with heterozygosity for primary carnitine deficiency can produce carnitine depletion and impaired oxidation of fatty acids.

In this study, we investigated the mechanism producing partial carnitine deficiency in heterozygotes by characterizing carnitine transport in fibroblasts and in the kidneys of parents of an affected patient. Our results indicate that increased urinary losses may be responsible for the partial reduction in plasma carnitine levels.

\section{MATERIALS AND METHODS}

\section{Subjects}

The proband, a white male, was born at term after an uneventful pregnancy to healthy, unrelated parents. A cesarean section was performed for breech presentation. His birth weight was $3.59 \mathrm{~kg}$ and he had a normal neonatal course. At 5 weeks of age, he started vomiting and having abdominal pain, which were ascribed to a milk allergy, and was switched to a protein hydrolysate formula (Alimentum). He had continuous episodes of upper respiratory tract infections that required therapy with multiple antibiotics and placement of myringotomy tubes in both ears at 7 months of age. At 8 months of age, during an upper respiratory tract infection, he became irritable and refused feedings. Subsequently, he became lethargic, prompting admission to a regional hospital for possible meningitis. During the hospital stay, he was lethargic and irritable, with findings consistent with bacterial otitis media and right lobar pneumonia. He also had hepatomegaly, hypoglycemia with low glucose levels in the cerebrospinal fluid, mild anemia, and hyperammonemia (up to $135 \mu \mathrm{M}$, normal: 9-33 $\mu \mathrm{M}$ ) with mildly elevated liver function tests. This suggested a diagnosis of Reye's syndrome. An electroencephalogram had abnormal results, suggesting diffuse disturbance of brain function with intact brainstem functioning. A computed tomography scan of the brain revealed prominence of the ventricular system. Biochemical evaluation indicated nonspecific abnormality of plasma and urine amino acids and the presence of dicarboxylic acids in the urine, interpreted as secondary to the presence of medium-chain triglycerides in the formula. He responded positively to intravenous fluids and glucose and was discharged home on a reduced protein formula because of the hyperammonemia. He was referred to a geneticist for a possible defect in the urea cycle.

Genetic evaluation at 9 months of age showed a child of appropriate weight and height, with normal developmental milestones, but slightly reduced muscle tone. Biochemical evaluation indicated persistent hyperammonemia, normal plasma amino acids and urine orotic acid. Plasma carnitine levels returned extremely low, with an apparently normal urinary carnitine excretion, which, however, was extremely elevated because of the low plasma carnitine levels (Table 1).

The child was given carnitine supplements that were gradually increased to $180 \mathrm{mg} / \mathrm{kg}$ per day. This
Table 1

Plasma and urine carnitine in a family with primary carnitine deficiency

\begin{tabular}{lccc}
\hline & $\begin{array}{c}\text { Plasma } \\
\text { carnitine } \\
(\mu \mathrm{M})\end{array}$ & $\begin{array}{c}\text { Urine } \\
\text { carnitine } \\
\text { (nmol/mg creatinine) }\end{array}$ & $\begin{array}{c}\text { Fractional loss } \\
\text { (\% of filtered } \\
\text { load) }\end{array}$ \\
\hline Proband & 1 & 216 & 92 \\
Mother & 21 & 128 & 5 \\
Father & 24 & 255 & 4.5 \\
Controls & $30-70$ & $60-400$ & $<5$ \\
\hline
\end{tabular}

Only total carnitine (free + acylcarnitine) is reported.

caused a progressive normalization of the hepatomegaly, the hyperammonemia, and the liver function tests. The parents also noted that the child became much more active and had a reduction in the frequency and severity of infections. Repeated carnitine determinations demonstrated normalization of plasma carnitine levels, with urinary carnitine excretion increasing 30 times above the normal range, suggesting a carnitine transport defect. At 21 months of age, a skin biopsy was obtained and defective carnitine transport was confirmed in fibroblasts.

Family history was significant for the absence of consanguinity. There was a history of allergy in the father and his brother. There was a strong history of heart disease on the side of the maternal grandmother whose aunt died at the age of 56 with a cardiomyopathy described as "unusual type" by the family cardiologist because it did not respond to conventional inotropic therapy. There was no history of hypertension or increased cholesterol levels in family members with heart problems.

Echocardiograms of both parents performed at ages 33 and 36 years in the mother and father, respectively, revealed structurally normal hearts with normal ejection fractions of $66 \%$ and $69 \%$, respectively.

\section{Methods}

Skin biopsies were obtained from the proband and both parents after receipt of informed consent to confirm the diagnosis of primary carnitine deficiency. Control fibroblasts and fibroblasts from a positive control with primary carnitine deficiency (GM10665) were obtained from the Coriell Institute for Medical Research, Camden, NJ. Fibroblasts were grown in Dulbecco's modified minimal essential medium supplemented with $15 \%$ fetal bovine serum.

Carnitine transport was measured with the cluster-tray method ${ }^{13}$ described previously for sugar and amino acid transport. ${ }^{14,15}$ Cells were seeded in 24-well plates (Costar, Acton, MA) and grown to confluence. The medium was replaced twice a week and 24 hours before the transport experiment. On the day of the experiment, cells were washed twice and incubated at $37^{\circ} \mathrm{C}$ in Earle's balanced salt solution containing $5.5 \mathrm{mM}$ D-glucose and supplemented with $1 \%$ bovine serum albumin for 90 minutes. This procedure was performed to deplete intracellular stores of carnitine and amino acids, to minimize possible trans-effects. ${ }^{16}$ Cells were then incubated for the indicated time in the presence of carnitine $(0.5 \mu \mathrm{M}, 0.5 \mu \mathrm{Ci} / \mathrm{mL})$. The transport reaction was stopped by rapidly washing the cells 4 times with ice-cold $0.1 \mathrm{M} \mathrm{MgCl}_{2}$. Intracellular water 
containing radioactive carnitine was extracted with $0.5 \mathrm{~mL}$ of ethanol, which was added to $4 \mathrm{~mL}$ of scintillation fluid and counted in a beta-counter (Beckmann LS 7500). The proteins inside each well of the 24-well plate were determined using a modified Lowry procedure. ${ }^{17}$ In parallel plates, intracellular water content was determined using the equilibrium distribution of 3-O-methyl-D-glucose, ${ }^{18}$ which is reached in 10 minutes in human fibroblasts. ${ }^{15}$ The average water content for all cell strains used in this study was $7.05 \pm 0.82 \mu \mathrm{L} / \mathrm{mg}$ of cell protein.

Kinetic analysis of carnitine transport was performed using nonlinear regression analysis of the data (after correction for nonsaturable uptake measured at $1-10 \mathrm{mM}$ cold carnitine) according to a Michaelis-Menten equation and parameters expressed as means $\pm 95 \%$ confidence intervals.

Plasma and urine carnitine were measured by a radiochemical method according to standard procedures. ${ }^{19}$ Urinary carnitine losses were measured for 2 hours with midpoint plasma sampling. The fractional loss of carnitine in the urine was measured by dividing urinary carnitine (in micromoles) for the amount of carnitine filtered during the urine collection time. Glomerular filtrate was estimated from creatinine clearance. Healthy volunteers, enrolled after the institutional review board's approval and informed consent (HIC No. 334-97), were similar to the parents in age (range, 23-48 years) and included six females and five males. They had no history of heart or kidney disease.

Linear regression was performed according to standard procedures using Excel, and significance was determined by analysis of variance.

\section{RESULTS}

Time course of carmitine accumulation by control and transport-defective fibroblasts

Figure 1 shows the accumulation of carnitine by human fibroblasts. In normal cells, total carnitine uptake (panel A) was linear up to 4 hours. Carnitine nonsaturable uptake, measured in the presence of $1 \mathrm{mM}$ cold carnitine (panel B), increased up to 15 minutes after which equilibrium (i.e., extracellular concentration) was approached and only a minimal increase in intracellular carnitine accumulation was observed. Normal fibroblasts had significant mediated carnitine accumulation (i.e., the difference between total uptake and uptake in the presence of $1 \mathrm{mM}$ cold carnitine) even at the shortest point tested ( $1 \mathrm{~min}$ ) (panel C). However, at this short time interval mediated transport was only about $50 \%$ of total transport. This fraction increased to 58,85 , and $93 \%$ of total transport at 15,60 , and 240 minutes, respectively.

In fibroblasts with defined carnitine transport deficiency (GM10665), there was no significant difference between carnitine transport in the absence or presence of $1 \mathrm{mM}$ cold carnitine. Carnitine transport from fibroblasts of our proband was similar to the positive control, confirming a diagnosis of primary carnitine deficiency.

\section{Kinetic constants for camitine transport by human fibroblasts}

Primary carnitine deficiency is transmitted as an autosomal recessive trait, and heterozygotes have partially reduced plasma carnitine levels and a partial impairment of carnitine transport. ${ }^{1}$ Therefore, we measured the kinetics of carnitine transport by fibroblasts from our patient and both of his parents (Fig. 2). Kinetic analysis showed marked impairment of saturable carnitine transport in the proband's fibroblasts and partially impaired carnitine transport in fibroblasts from both parents, whose cells retained a normal $K_{\mathrm{m}}$ toward carnitine $(6-9 \mu \mathrm{M})$ but reduced $V_{\max }$ (Fig. 2A). The partial impairment of carnitine transport in both parental cells further confirmed the autosomal recessive inheritance of this condition.

Although it is well known that fibroblasts and tissues from homozygous patients have reduced accumulation of carnitine, there are limited data on the effect of heterozygosity (i.e., the presence of only one functional gene for the carnitine transporter) on carnitine accumulation in cells or tissues. ${ }^{20}$ Therefore, we examined a timecourse of carnitine accumulation to determine whether, at equilibrium, cells from the heterozygous parents accumulated the same amount of carnitine as controls. Figure $2 \mathrm{~B}$ shows that carnitine reached equilibrium after about 30 hours in control cells when the carnitine gradient (in/out) became about 80 . By contrast, cells homozygous for the carnitine transporter defect had minimal carnitine accumulation even after 54 hours. Cells from both heterozygous parents reached an apparent equilibrium after 30 hours, but the level of carnitine inside the cell was about one half of that of control cells.

\section{Urinary camitine losses in heterozygotes for primary camitine deficiency}

Table 1 reports plasma and urinary carnitine in the patient and in his parents at diagnosis. Urinary carnitine (in $\mathrm{nmol} / \mathrm{mg}$ of creatinine) seemed normal both in patient and parents. However, after calculating the amount of carnitine filtered during urine collection and the fraction of carnitine lost in the urine, results revealed that the proband lost more than $90 \%$ of filtered carnitine whereas both parents' losses were in the high normal range. For other types of kidney transporters, such as the Na-glucose symporter defective in renal glycosuria, glucose is lost in the urine when the filtered load exceeds the capacity for tubular resorption. ${ }^{21}$ The filtered load increases with the plasma concentration of the solute. Therefore, we plotted the urinary losses of carnitine as a function of plasma carnitine levels to determine whether there was an increase in urinary losses with the increase in plasma concentrations (Fig. 3). These studies were performed with different control subjects and without increasing carnitine concentration in the same subject, explaining the scatter of the data obtained. There was a statistically significant $(P<0.05)$ increase in the urinary loss of carnitine in normal subjects as the plasma concentration of carnitine increased.

To compensate for the physiological increase in urinary losses expected at higher plasma concentrations, we divided the fractional carnitine loss (as percent of filtered load) for the plasma carnitine concentration (in $\mathrm{mM}$ ) in controls and both parents (panel B). After this normalization, urinary carnitine losses by both parents were two to three times those of controls and differed significantly $(P<0.01)$ from the normal range.

\section{DISCUSSION}

Primary carnitine deficiency, first reported in 1973,22 is an autosomal recessive disorder caused by 
impaired carnitine transport. The defect affects renal tubular epithelial cells and is also expressed in fibroblasts. ${ }^{2,3}$ This disorder does not seem to affect the liver whose carnitine stores are normalized to near normal after carnitine supplementation, in contrast with the muscles which remain carnitine depleted. ${ }^{2}$ In this study, we characterize carnitine transport and accumulation in heterozygotes for primary carnitine deficiency.

Fibroblasts from our patients failed to accumulate carnitine, resembling cells from another patient with the same syndrome (Fig. 1). Kinetic analysis revealed absent saturable carnitine transport in fibroblasts from the proband and reduced $V_{\max }$ for carnitine transport in cells from both parents (Fig. $2 \mathrm{~A}$ ). The $50 \%$ reduction in $V_{\max }$ is consistent with a reduced number of membrane carnitine transporters in fibroblasts from both heterozygous parents and has been reported previously in other families. ${ }^{2,3}$ However, it was unclear whether the reduced number of membrane transporters had any effect on intracellular carnitine content. Our data show that fibroblasts from both parents had a reduced ability to concentrate carnitine reaching only half of the steady-state levels of control cells (Fig. 2B). The mechanism producing a decreased steady-state level of carnitine in fibroblasts from heterozygous parents is probably related to the decreased $V_{\max }$ for carnitine transport. When a specific gradient is reached in heterozygous cells, the efflux of carnitine through diffusion and other less specific transporters equals the decreased influx caused by the partially reduced number of transporters.

Heterozygotes for primary carnitine deficiency have partially reduced plasma carnitine levels (Table 1). The mechanism at the basis of this phenomenon is unclear because common measurement of urinary carnitine excretion and its normalization to creatinine cannot distinguish heterozygotes from controls (Table 1). In healthy individuals, urinary carnitine losses increased significantly with elevation of plasma

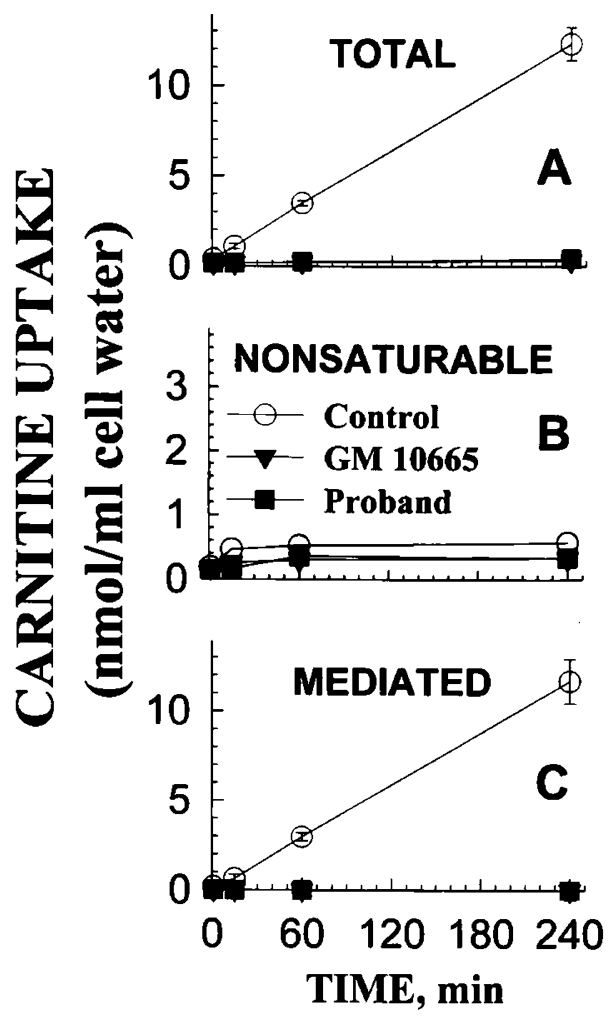

Fig. 1 Time course of carnitine accumulation by normal and transport-defective human fibroblasts. Confluent cells were washed and incubated for 90 minutes in Earle's balanced salt solution containing $\mathrm{D}$-glucose $(5.5 \mathrm{mM})$ and supplemented with $1 \%$ bovine serum albumin. Cells were then incubated for the indicated times with ${ }^{3} \mathrm{H}$-carnitine $(0.5 \mu \mathrm{M}, 0.5 \mu \mathrm{Ci} / \mathrm{mL})$ in the absence (panel $\mathrm{A}$ ) or presence (panel $B$ ) of $1 \mathrm{mM}$ cold carnitine, included to assess nonsaturable carnitine uptake. Mediated transport (panel C) was obtained by subtracting the nonsaturable component from total transport. Each point is the mean of triplicates $\pm \mathrm{SD}$.
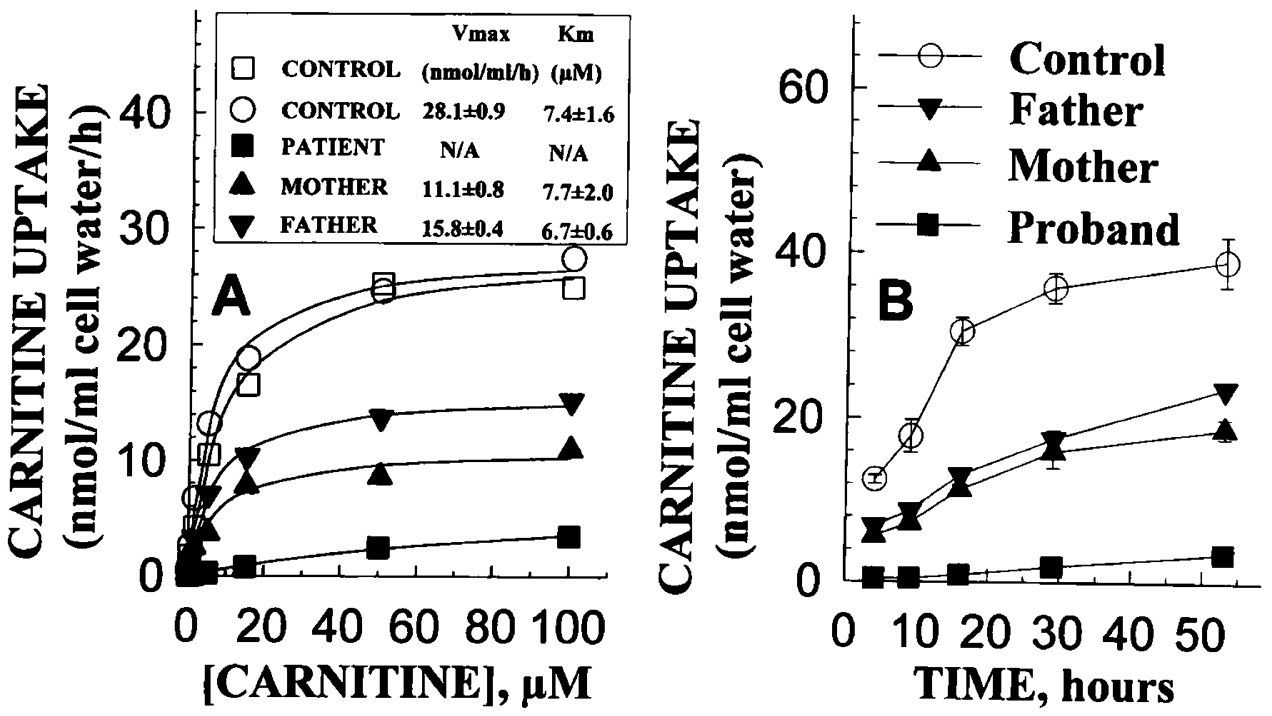

Fig. 2 Carnitine transport by fibroblasts from a family with primary carnitine deficiency. (A) Kinetic analysis of carnitine transport in fibroblasts from the proband and his parents. Note the more severe impairment of carnitine transport in the mother's compared with the father's fibroblasts. Points are means of triplicates and are corrected for nonspecific uptake (measured in the presence of $10 \mathrm{mM}$ cold carnitine). Lines represent the best fit of data to a Michaelis-Menten equation. (B) Time course of carnitine $(0.5 \mu \mathrm{M})$ accumulation by human fibroblasts. Points are means \pm SD of triplicates. 

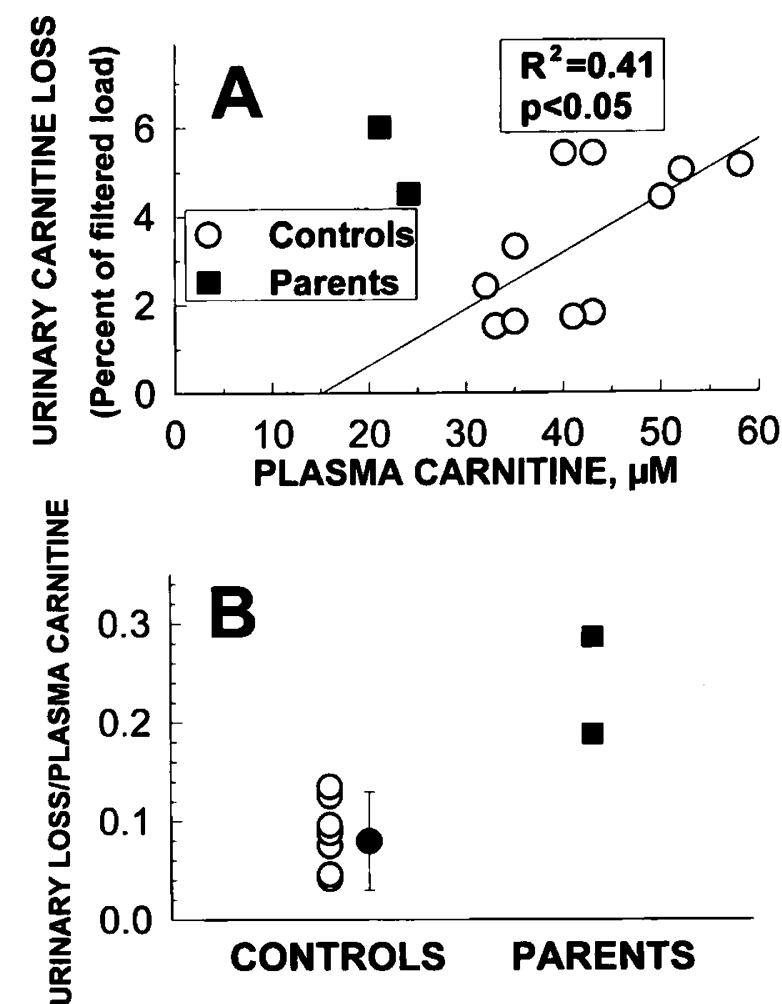

Fig. 3 Urinary carnitine losses as a function of plasma carnitine concentration in normal subjects and in heterozygotes for primary carnitine deficiency. (A) Urine was collected for 2 hours and plasma creatinine and carnitine levels were determined at midpoint. Controls were apparently healthy individuals (ages, 23-48 years) with no history of renal or heart disease. The fraction of carnitine lost was calculated by dividing the amount of urinary carnitine by the amount of carnitine filtered by the kidney in the 2 hours of the collection (obtained from creatinine clearance). (B) Fractional losses of carnitine in the urine (percent) were normalized for the plasma carnitine level $(\mu \mathrm{M})$. For controls, the average is shown to the right of individual points with the $99 \%$ confidence interval.

carnitine levels (Fig. 3A). When the fraction of carnitine lost in the urine was calculated and normalized to plasma carnitine levels, heterozygotes had indeed increased urinary carnitine losses (Fig. 3B). This need for normalization indicates that at the same plasma carnitine levels, heterozygotes have increased urinary losses compared with controls. In renal glycosuria, two different types have been defined based on a decreased threshold for excretion (type $A$ ) or on decreased maximal capacity of resorption (type B). ${ }^{21}$ Formal renal titration curves are needed to define which one of these two mechanisms impairs carnitine resorption in heterozygotes for primary carnitine deficiency. In addition to the renal mechanism, we cannot exclude the contribution of increased intestinal losses to partial carnitine deficiency. ${ }^{11}$ Although fecal carnitine excretion was determined in one study, ${ }^{23}$ its value is unclear because of the transformation of carnitine by Escherichia coli and other intestinal bacteria, which, unlike mammalian cells, have the capacity to metabolize carnitine. ${ }^{24}$

The lower capacity to accumulate carnitine compounded with the reduced plasma carnitine levels may lead to reduced availability of intracellular carnitine and pose a risk for heterozygotes for tissue carnitine deficiency. In physiological conditions, carnitine palmityltransferase- 1 is the rate-limiting step for fatty acid oxidation. It is possible that the reduced levels of carnitine may become rate limiting in conditions such as fasting when carnitine palmityl- transferase- 1 is fully stimulated. In these conditions, the limited capacity to oxidize fatty acids during starvation may have some effects on heart or muscle function in heterozygotes. Transient cardiomyopathy, possibly precipitated by recurrent attacks of vomiting and consequent starvation, has been described in the heterozygous brother of a patient with primary carnitine deficiency. ${ }^{9}$ Less information is available concerning the role of heterozygosity for carnitine deficiency in adult-onset heart disease. The family history of our patients is intriguing because cardiomyopathy and heart disease, not explained by common risk factors, were prevalent in the maternal side of the pedigree. Echocardiograms performed in both parents (ages, 33 and 36 years) with partial carnitine deficiency were normal, indicating that modest reductions in carnitine levels do not affect heart function. However, we cannot exclude the possibility that heterozygosity for the carnitine transport defect may contribute to more severe carnitine deficiency when a suboptimal diet or a concomitant ailment is superimposed. Therefore, further studies are needed to define whether partial carnitine deficiency contributes to the increased incidence of heart disease in this family.

\section{References}

1. Pons R, De Vivo DC. Primary and secondary carnitine deficiency. J Child Neurol Suppl 1995;10:\$82-S24.

2. Treem WR, Stanley CA, Finegold DN, Hale DE, Coates PM. Primary carnitine deficiency due to a failure of carnitine 
transport in kidney, muscle, and fibroblasts. $N$ Engl J Med 1988;319:1331-1336.

3. Eriksson BO, Gustafson B, Lindstedt S, Nordin I. Transport of carnitine into cells in hereditary carnitine deficiency. Inherit Metab Dis 1989;12:108-111.

4. Stanley CA, Deleeuw S, Coates PM, Vianay LC, Divry P, Bonnefont IP, et al. Chronic cardiomyopathy and weakness or acute coma in children with a defect in carnitine uptake. Ann Neurol 1991;30:709-716.

5. Tein I, De Vivo CD, Bierman F, Pulver P, De Meirler LJ, Cvitanovic-Sojat L, et al. Impaired skin fibroblast carnitine uptake in primary systemic carnitine deficiency manifested by childhood carnitine-responsive cardiomyopathy. Pediatr Res 1990;28:247-255.

6. Waber LJ, Valle D, Neill C, DiMauro S, Shug A. Carnitine deficiency presenting as familial cardiomyopathy: A treatable defect in carnitine transport. J Pediatr 1982;101:700-704.

7. Bennett MJ, Hale DE, Pollitt RJ. Stanley CA, Variend S. Endocardial fibroelastosis and primary carnitine deficiency due to a defect in the plasma membrane carnitine transporter. Clin Cardiol 1996;19:243-246.

8. Christodolou J, Teo SH, Hammond J, Sim KG, Hsu BYL, Stanley $\mathrm{CA}$, et al. First prenatal diagnosis of the carnitine transporter defect. Am J Med Genet 1996;66:21-24.

9. Garavaglia B, Uziel G, Dworzak F, Carrara F, Di Donato S Primary carnitine deficiency: Heterozygote and intrafamilial variation. Neurology 1991;41:1691-1693.

10. Rebouche CJ, Engel AG. Kinetic compartmental analysis of carnitine metabolism in the human carnitine deficiency syndromes. J Clin Invest 1984;73:857-867.

11. Rashed MS, Ozand PT, Bennett MJ, Barnard J], Govindaraju DR, Rinaldo P. Diagnosis of inborn errors of metabolism in sudden death cases by acylcarnitine analysis of post-mortem bile. Clin Chem 1995;41:1109-1114.

12. Rinaldo P, Stanley CA, Hsu BYL, Sanchez LA, Stern HJ. Sudden neonatal death in carnitine transporter deficiency.
J Pediatr 1997;131:304-305.

13. Gazzola GC, Dall'Asta V, Franchi-Gazzola R, White MF. The cluster-tray method for rapid measurement of solute fluxes in adherent cultured cells. Anal Biochem 1981;115:368-374.

14. Longo N, Franchi-Gazzola R, Bussolati $O$, Dall'Asta V, Foa PP, Guidotti GG, et al. Effect of insulin on the activity of amino acid transport systems in cultured human fibroblasts. Biochim Biophys Acta 1985;844:216-223.

15. Longo N, Griffin LD, Elsas LJ. Influx and efflux of 3-O-methylD-glucose by cultured human fibroblasts. Am J Physiol 1988:254:C628-C633.

16. Gazzola GC, Dall'Asta V, Guidotti GG. The transport of neutral amino acids in cultured human fibroblasts. J Biol Chem 1980;255:929-936.

17. Wang CS, Smith RL. Lowry determination in the presence of Triton X-100. Anal Biochem 1975;63:414-417.

18. Kletzien RF, Pariza MW, Becker JE, Potter VR. A method using 3-O-methyl-D-glucose and phloretin for the determination of intracellular water space. Anal Biochem 1975;68:537-544.

19. Hoppel CL. Determination of carnitine. In: Hommes FA, editor. Techniques in diagnostic human biochemical genetics. New York: Wiley-Liss, 1991:309-326.

20. Eriksson BO, Lindstedt S, Nordin I. Hereditary defect in carnitine membrane transport is expressed in fibroblasts. Eur J Pediatr 1988;147:662-668.

21. Elsas LJ, Longo N. Glucose transporters. Annu Rev Med 1992;43:377-393.

22. Engel AG, Angelini C. Carnitine deficiency of human skeletal muscle with associated lipid storage myopathy. Science 1973;179:899-902.

23. Rodriguez-Pereira R, Scholte HR, Lyuyt-Houwen IEM, Vaavdrager-Verduin MHM. Cardiomyopathy associated with carnitine loss in kidney and small intestine. Eur J Pediatr 1988;148:193-197.

24. Kleber HP. Bacterial carnitine metabolism. FEMS Microbiol Lett 1997;147:1-9. 